\title{
Accelerated partial breast irradiation utilizing brachytherapy: patient selection and workflow
}

\author{
Chirag Shah, MD', Jessica Wobb, MD², Bindu Manyam, MD', Atif Khan, MD³, Frank Vicini, MD4 \\ 'Department of Radiation Oncology, Taussig Cancer Institute, Cleveland Clinic, Cleveland, Ohio, ${ }^{2}$ Department of Radiation Oncology, \\ Ohio State University, Columbus, Ohio, ${ }^{3}$ Department of Radiation Oncology, Robert Wood Johnson University Hospital/Cancer Institute \\ of New Jersey, New Brunswick, New Jersey, ${ }^{4} 2{ }^{\text {st }}$ Century Oncology, Michigan Healthcare Professionals, Farmington Hills, Michigan, USA
}

\begin{abstract}
Accelerated partial breast irradiation (APBI) represents an evolving technique that is a standard of care option in appropriately selected woman following breast conserving surgery. While multiple techniques now exist to deliver APBI, interstitial brachytherapy represents the technique used in several randomized trials (National Institute of Oncology, GEC-ESTRO). More recently, many centers have adopted applicator-based brachytherapy to deliver APBI due to the technical complexities of interstitial brachytherapy. The purpose of this article is to review methods to evaluate and select patients for APBI, as well as to define potential workflow mechanisms that allow for the safe and effective delivery of APBI. Multiple consensus statements have been developed to guide clinicians on determining appropriate candidates for APBI. However, recent studies have demonstrated that these guidelines fail to stratify patients according to the risk of local recurrence, and updated guidelines are expected in the years to come. Critical elements of workflow to ensure safe and effective delivery of APBI include a multidisciplinary approach and evaluation, optimization of target coverage and adherence to normal tissue guideline constraints, and proper quality assurance methods.
\end{abstract}

Key words: APBI, brachytherapy, breast cancer, breast conserving therapy, radiation therapy.

\section{Purpose}

Breast conserving therapy, consisting of lumpectomy followed by 6 to 7 weeks of whole breast irradiation (WBI), has been shown in multiple randomized trials to produce equivalent clinical outcomes compared to mastectomy, as well as reduced breast cancer mortality when compared to lumpectomy alone [1,2,3,4]. However, the protracted nature of treatment can pose financial and logistical hardships on patients and medical systems $[5,6,7]$. Therefore, there has been increased interest in developing shorter treatment courses without compromising treatment efficacy, in particular local control. One such technique is accelerated partial breast irradiation (APBI), which covers the area surrounding the lumpectomy cavity with a margin and typically is completed in one week or less [8]. Multiple studies have compared APBI with WBI and have found equivalent rates of local control and thus, APBI has emerged as a viable, safe treatment option for properly selected patients with early stage breast cancer $[9,10,11,12,13]$. As interest in and the utilization APBI grows, a standardized approach to treatment and workflow is necessary for many groups. The purpose of this review is to provide clinicians with a guide on patient se- lection and treatment workflow for patients undergoing brachytherapy-based APBI.

\section{Discussion}

\section{Patient selection}

Society-specific guidelines have utilized a combination of data from patients treated with APBI, data from patients treated with WBI including patterns of failure, and expert opinion to define appropriateness criteria for the utilization of APBI off-protocol (Table 1) [14,15,16,17]. The ASTRO consensus statement, published in 2009, is one of the most commonly utilized guidelines and uses clinical and pathologic features that stratify patients into suitable, cautionary, and unsuitable candidates for the use of APBI [16]. However, several studies have failed to show a correlation between local failure and consensus groupings $[18,19,20]$. Nevertheless, since the publications of the ASTRO consensus guidelines, data has demonstrated an increase in the use of APBI for suitable candidates and a decline for those that were unsuitable, demonstrating that published guidelines were being adopted by radiation oncologists [21]. A more recent guideline from the
Address for correspondence: Chirag Shah, MD, Department of Radiation Oncology, Taussig Cancer Institute, Cleveland Clinic, 9500 Euclid Avenue, T28, Cleveland, Ohio 44195, USA, phone: +1 248217 7810, fax: +1 216904 7950, $₫$ e-mail: csshah27@hotmail.com
Received: 16.01 .2016

Accepted: 08.02 .2016

Published: 26.02.2016 
American Brachytherapy Society has incorporated much of the recent APBI data and provides a simpler set of guidelines for clinicians with the ASTRO guidelines to be updated in 2016. In the interim, clinicians can utilize the consensus guidelines available or eligibility criteria from the four randomized trials of APBI that have shown no difference in local recurrence or other clinical outcomes as compared with WBI (Table 1) $[9,10,14,15,16,17]$.

\section{Workflow}

Interstitial brachytherapy is a technically complex procedure that is performed at a limited number of centers. Therefore, the discussion of workflow will focus on the utilization of applicator-based brachytherapy, which is more routinely used.

\section{Pre-operative assessment}

Patients interested in APBI should be evaluated by a radiation oncologist and medical oncologist prior to surgery. Multidisciplinary evaluation establishes optimal sequencing and identifies patients who may or may not be appropriate for APBI $[14,15,16,17]$. With regards to surgical planning, tumor location (ex. upper inner quadrant tumors) can present challenges with applicator brachytherapy. However, recent innovations in applicator technology allow for delivery to cavities with thin skin bridges ( $<7 \mathrm{~mm})$, elliptical shape, and difficult locations, all while maintaining target coverage and sparing organs at risk $[22,23,24]$. For patients considering oncoplastic procedures, limited data are available at this time regarding APBI and oncoplastic surgery and clinicians should consider performing such cases on protocol. It is important to establish follow up and a treatment schedule pre-operatively to avoid scenarios such as leaving an applicator in over 2 weekends and to avoid delays in initiating therapy.

\section{Post-operative}

Following surgery and prior to proceeding with brachytherapy, it is important to review the pathology report for tumor size, histology, margins, nodal status, lymphovascular space invasion (LVSI), and receptor status to ensure appropriate patient selection $[14,15,16,17]$. Applicators are typically placed by the surgeon postoperatively, though they can be placed by radiation oncologists under ultrasound guidance as well using a closed cavity technique. Some centers utilize cavity evaluation devices or prep catheters that are placed in the operating room and are switched out with a treatment applicator following review of pathology. On the other hand, some centers will not place any device at the time of surgery and will place the applicator postoperatively using a percutaneous ultrasound guided approach. Once the applicator is placed, patients can undergo computed tomography (CT) scan for planning. At some centers, a CT scan is performed prior to the placement of the applicator, though this is not required. At the time of simulation, some centers will place contrast if using a balloon device. Computed tomography scan should be performed with slice thickness of $5 \mathrm{~mm}$ or less. At the time of simulation, it is important to evaluate applicator/tissue conformance with the cavity, as less than $10 \%$ of the PTV_EVAL should be trapped air/fluid [25]. With regards to wound care, the site should be cleaned at each fraction with betadine/

Table 1. Consensus statement guidelines and eligibility criteria

\begin{tabular}{|c|c|c|c|c|c|c|}
\hline & ASTRO & ABS & GEC-ESTRO & ASBS & GEC-ESTRO Trial & $\begin{array}{c}\text { National } \\
\text { Institute of } \\
\text { Oncology Trial }\end{array}$ \\
\hline & \multicolumn{4}{|c|}{ Consensus statements } & \multicolumn{2}{|c|}{ Eligibility criteria } \\
\hline Age & $\geq 60$ years & $\geq 50$ years & $\geq 50$ years & $\geq 45$ years & $\geq 40$ years & \\
\hline Size & pT1 & $\begin{array}{l}\text { pTis, pT1-2 } \\
(\leq 3 \mathrm{~cm})\end{array}$ & $\begin{array}{c}\text { pT1-2 } \\
(\leq 3 \mathrm{~cm})\end{array}$ & $\begin{array}{l}\text { pTis, pT1-2 } \\
(\leq 3 \mathrm{~cm})\end{array}$ & $\begin{array}{l}\text { pTis, pT1-2a } \\
(\leq 3 \mathrm{~cm})\end{array}$ & pT1 \\
\hline Nodal status & pNO & pNO & pNO & pNO & pNO & $\mathrm{cNO} / \mathrm{pNO} / \mathrm{pN} 1 \mathrm{mi}$ \\
\hline Histology & IDC/Favorable & IDC/ILC/DCIS & IDC & IDC/DCIS & $\begin{array}{c}\text { DCIS }(\mathrm{VNPI}<8) / \\
\text { IDC/ILC }\end{array}$ & IDC \\
\hline Margins & Negative & Negative & Negative & Negative & Negative & Negative \\
\hline $\begin{array}{l}\text { Estrogen } \\
\text { receptor }\end{array}$ & Positive & Any & Any & - & - & - \\
\hline LVSI & Negative & Negative & Negative & - & Negative & - \\
\hline $\begin{array}{l}\text { Systemic } \\
\text { therapy }\end{array}$ & No neoadjuvant & - & No neoadjuvant & - & - & - \\
\hline $\begin{array}{l}\text { Endocrine } \\
\text { therapy }\end{array}$ & - & - & - & - & - & - \\
\hline Grade & Any & - & Any & - & - & Grade 1-2 \\
\hline
\end{tabular}

ASTRO - American Society for Radiation Oncology, ABS - American Brachytherapy Society, GEC-ESTRO - Groupe Européen de Curiethérapie, ASBS - American Society of Breast Surgeons, IDC - invasive ductal carcinoma, ILC - invasive lobular carcinoma, DCIS - ductal carcinoma in situ, LVSI - lymphovascular space invasion 
antiseptic. Antibiotics can be prescribed and are usually done based on surgeon preference with limited data for or against the practice. Patients are advised against taking a shower for the duration of treatment.

\section{Planning}

Multiple systems are available for planning; however, it is important to follow consistent guidelines with respect to target volume coverage and organs at risk. Table 2 provides several sets of guidelines with respect to both. NSABP B39 recommends the cavity be outlined with the CTV = PTV. The PTV_EVAL is defined as the breast tissue included in a $10 \mathrm{~mm}$ expansion of the balloon volume limited to $5 \mathrm{~mm}$ from the skin surface and posterior breast [25]. The prescription is $34 \mathrm{~Gy}$ in 10 fractions delivered twice daily. It is recommended that $95 \%$ of the PTV_EVAL receive at least $90 \%$ of the prescribed dose ( $<10 \%$ trapped air/fluid) with $V_{150}$, and $V_{200}$ being $<50$ cc and $<10 \mathrm{cc}$, respectively [25]. Additional constraints include $<60 \%$ of the whole breast receiving $\geq 50 \%$ of the prescription and a maximal skin dose of $145 \%$ of the prescription [25]. It should be noted that these criteria were designed for single lumen applicators and with the advent of new applicators, improved volume coverage, skin sparing, and reduced dose to the chest wall can be obtained $[22,23,24]$. The maximum dose to skin should be less than $100 \%$ and chest wall constraints are recommended $(<100 \%$ of prescription). It should be noted that with strut applicators there is the potential for higher volumes of $\mathrm{V}_{200}$ in the target volume, similar to interstitial implants [22].

\section{Quality assurance}

Multiple methods exist to implement quality assurance (QA) for brachytherapy based APBI; however, it is important that individual centers utilize consistent QA methods and metrics. Imaging should be performed prior to each fraction and compared to imaging from simulation; with balloon based applicators this is important to confirm that the balloon has not been deflated or lost volume [25]. Multiple strategies exist including CT, ultrasound/X-ray with some centers using $\mathrm{CT}$ for one fraction each day and an ultrasound/X-ray for the second. Alternatively, some centers use ultrasound/X-ray at each fraction. Assuring the appropriate length of the applicator is another important
QA metric. This can be verified on imaging but can also be verified by measuring the length of applicator from the tip to the skin surface prior to each fraction. Similarly, with multilumen and strut based devices where isodose distributions are not uniform but rather shaped, rotation must be evaluated prior to each fraction; this can be done by making a mark on the skin and one on the applicator (disk attached to applicator) [25].

\section{Completion/follow up}

At the completion of brachytherapy, the applicator should be removed. The insertion site can be closed with steri-strips or a stitch can be placed. Establishing longterm follow up is imperative to assess not only for oncologic outcomes but toxicity and quality of life as well. Proper follow up also facilitates the transition from the treatment phase to the survivorship phase for the patient. Initial follow up is typically performed one week after completion of brachytherapy to assess for infections, catheter related complications, or other acute toxicities. Following this, patients can be seen every 3-6 months for the first few years at which point visits can be spaced further apart depending on frequency of follow up with surgery and medical oncology [26]. At each visit, it is important to document any acute, subacute, and late toxicities. Follow up mammography should be ordered as recommended with no change in follow up imaging recommendations for patients undergoing brachytherapy as compared with external beam radiotherapy.

\section{Future directions}

Brachytherapy based APBI continues to evolve at this time. With the advent of multi-lumen and strut based devices, a larger number of patients are able to receive APBI with improved target coverage and reduced dose to organs at risk $[22,23,24]$. Treatment schedules continue to evolve and shorten with two day fractionation being evaluated at this time [27]. Additional brachytherapy strategies including low-dose-rate (LDR) permanent seed implants are being evaluated as well [28]. While other partial breast techniques have been studied (ex. intraoperative radiotherapy), due to higher rates of local recurrence and short follow up, these techniques should not be considered comparable to APBI and remain investigational and not recommended for use off-protocol $[29,30]$.

Table 2. Target volume and organ at risk constraints for applicator APBI

\begin{tabular}{lccccc} 
& B39 Interstitial & B39 MammoSite & Contura Registry & SAVI & Recommendations \\
\hline $\mathrm{D}_{90}$ & $>90 \%$ & $>90 \%$ & - & $>90 \%$ & $\geq 95 \%$ \\
\hline Breast $\mathrm{V}_{150}$ & $\leq 70 \mathrm{cc}$ & $\leq 50 \mathrm{cc}$ & $\leq 50 \mathrm{cc}$ & $\leq 50 \mathrm{cC}$ & $\leq 50 \mathrm{cC}$ \\
\hline Breast $\mathrm{V}_{200}$ & $\leq 20 \mathrm{cc}$ & $\leq 10 \mathrm{cc}$ & $\leq 10 \mathrm{cc}$ & $\leq 20 \mathrm{cC}$ & $\leq 10 \mathrm{cC}$ \\
\hline Skin $\mathrm{D}_{\max }$ & $\leq 100 \%$ & $\leq 145 \%$ & $\leq 100 \%$ & $\leq 100 \%$ & $<100 \%$ \\
\hline Chest wall $\mathrm{D}_{\max }$ & - & - & $<145 \% \mathrm{Rx}$ & $\leq 100 \%$ & $<100 \%$
\end{tabular}

SAVI - strut-adjusted volume implant, $D_{90}$ - the percentage of the prescribed dose received by $90 \%$ volume of the prostate, $V_{150}-$ the percentage of the prostate volume receiving $150 \%$ of the prescribed dose or more, $V_{200}$ - the percentage of the prostate volume receiving $200 \%$ of the prescribed dose or more, $D_{\text {max }}-$ maximum dose, $\mathrm{Cc}-\mathrm{cm}^{3}$ 


\section{Conclusions}

1. Accelerated partial breast irradiation represents a standard of care for appropriately selected patients with early stage breast cancer. Key points to be taken from this technical review include:

2. Patient selection - clinicians evaluating patients for APBI off - protocol should use consistent guidelines, which may include consensus statements or eligibility criteria from published randomized studies.

3. Multidisciplinary management - allowing for radiation oncologists to evaluate the patient prior to surgery permits for optimization of patient selection, workflow, and appropriate APBI technique selection.

4. Dose volume histogram criteria should be utilized in order to maximize local control while limiting the potential for toxicity.

5. Consistent quality assurance measures allows for the delivery of safe treatment for each patient at each fraction; every program utilizing brachytherapy based APBI should have a set of quality assurance measures in place based on current standards and guidelines.

\section{Disclosure}

Authors report no conflict of interest.

\section{References}

1. Fisher B, Anderson S, Bryant J et al. Twenty-year follow-up of a randomized trial comparing total mastectomy, lumpectomy, and lumpectomy plus irradiation for the treatment of invasive breast cancer. N Engl J Med 2002; 347: 1233-1241.

2. Veronesi U, Cascinelli N, Mariani L et al. Twenty-year follow-up of a randomized study comparing breast-conserving surgery with radical mastectomy for early breast cancer. N Engl J Med 2002; 347: 1227-1232.

3. Litière $S$, Werutsky G, Fentiman IS et al. Breast conserving therapy versus mastectomy for stage I-II breast cancer: 20 year follow-up of the EORTC 10801 phase 3 randomized trial. Lancet Oncol 2012; 13: 412-419.

4. Early Breast Cancer Trialists' Collaborative Group (EBCTCG), Darby S, McGale P, Correa C et al. Effect of radiotherapy after breast-conserving surgery on 10-year recurrence and 15 year breast cancer death: meta-analysis of individual patient data for 10,801 women in 17 randomized trials. Lancet 2011; 378: $1707-1716$

5. Athas WF, Adams-Cameron M, Hunt WC et al. Travel distance to radiation therapy and receipt of radiotherapy following breast-conserving surgery. J Natl Cancer Inst 2000; 92: 269-271.

6. Stafford D, Szczys R, Becker R et al. How breast cancer treatment decisions are made by women in North Dakota. Am J Surg 1998; 176: 515-519.

7. Morrow M, White J, Moughan J et al. Factors predicting the use of breast-conserving therapy in stage I and II breast carcinoma. J Clin Oncol 2001; 19: 2254-2262.

8. Vicini F, Kestin L, Goldstein N. Defining the clinical target volume for patients with early-stage breast cancer treated with lumpectomy and accelerated partial breast irradiation: a pathologic analysis. Int J Radiat Oncol Biol Phys 2004; 60: $722-730$

9. Polgár C, Major T, Fodor J et al. Accelerated partial breast irradiation using high-dose-rate interstitial brachytherapy: 12-year update of a prospective clinical study. Radiother Oncol 2010; 94: 274-279.
10. Strnad V, Ott OJ, Hildebrandt G et al. 5-year results of accelerated partial breast irradiation using sole interstitial multicatheter brachytherapy versus whole-breast irradiation with boost after breast-conserving surgery for low-risk invasive and in-situ carcinoma of the female breast: a randomized, phase 3, non-inferiority trial. Lancet 2016; 387: 229-238.

11. Livi L, Meattini I, Marrazzo L et al. Accelerated partial breast irradiation using intensity-modulated radiotherapy versus whole breast irradiation: 5 -year survival analysis of a phase 3 randomized controlled trial. Eur J Cancer 2015; 51: 451-463.

12. Rodríguez N, Sanz X, Dengra J et al. Five-year outcomes, cosmesis, and toxicity with 3-dimensional conformal external beam radiation therapy to deliver accelerated partial breast irradiation. Int J Radiat Oncol Biol Phys 2013; 87: 1051-1057.

13. Shah C, Antonucci J, Wilkinson J et al. Twelve-year clinical outcomes and patterns of failure with accelerated partial breast irradiation versus whole-breast irradiation: results of a matched-pair analysis. Radiother Oncol 2011; 100: 210-214.

14. The American Society of Breast Surgeons. Consensus Statement for Accelerated Partial Breast Irradiation. American Society of Breast Surgeons Web site; available at: https:/ / www. breastsurgeons.org/statements/PDF_Statements/APBI.pdf. Updated August 15, 2012 [accessed September 10, 2015).

15. Shah C, Vicini F, Wazer D et al. The American Brachytherapy Society consensus statement for accelerated partial breast irradiation. Brachytherapy 2013; 12: 267-277.

16. Smith B, Arthur D, Buchholz T et al. Accelerated partial breast irradiation consensus statement from the American Society for Radiation Oncology (ASTRO). Int J Radiat Oncol Biol Phys 2009; 74: 987-1001.

17. Polgár C, Van Limbergen E, Pötter R et al. Patient selection for accelerated partial-breast irradiation (APBI) after breast conserving surgery: recommendations of the Groupe European de Curietherapie - European Society for Therapeutic Radiology and Oncology (GEC-ESTRO) breast cancer working group based on clinical evidence (2009). Radiother Oncol 2010; 94: 264-273.

18. Shaitelman S, Vicini F, Beitsch P et al. Five-year outcome of patients classified using American Society for Radiation Oncology consensus statement guidelines for the application of accelerated partial breast irradiation: an analysis of patients treated on the American Society of Breast Surgeons MammoSite Registry Trial. Cancer 2010; 116: 4677-4685.

19. Christoudias M, Collett A, Stull T et al. Are the American Society for Radiation Oncology guidelines accurate predictors of recurrence in early stage breast cancer patients treated with balloon-based brachytherapy? Int J Surg Oncol 2013; 2013: 829050.

20. Vicini F, Arthur D, Wazer D et al. Limitations of the American Society of Therapeutic Radiology and Oncology Consensus guidelines on the use of accelerated partial breast irradiation. Int J Radiat Oncol Biol Phys 2011; 79: 977-984.

21. Husain Z, Lloyd S, Shah C et al. Changes in brachytherapy-based APBI patient selection immediately before and after publication of the ASTRO consensus statement. Brachytherapy 2015; 14: 490-495.

22. Yashar CM, Scanderberg D, Kuske R et al. Initial clinical experience with the strut-adjusted volume implant (SAVI) breast brachytherapy device for accelerated partial-breast irradiation (APBI): first 100 patients with more than 1 year follow-up. Int J Radiat Oncol Biol Phys 2011; 80: 765-770.

23. Brown S, McLaughlin M, Pope D et al. A dosimetric comparison of the multilumen balloon breast brachytherapy catheter vs. the single-lumen MammoSite balloon device in patients treated with accelerated partial breast irradiation at a single institution. Brachytherapy 2011; 10: 68-73. 
24. Shah C, Ghilezan M, Arthur D et al. Initial clinical experience with multilumen brachytherapy catheters for accelerated partial breast irradiation. Brachytherapy 2012; 11: 369-373.

25. RTOG. Clinical Trials, Study Number 0413; available at: https://www.rtog.org/ClinicalTrials/ProtocolTable/ StudyDetails.aspx?study=0413 [retrieved February 8, 2016].

26. NCCN Clinical Practice Guidelines in Oncology: Breast Cancer; available at: http://www.nccn.org/professionals/physician_gls/pdf/breast.pdf [retrieved February 8, 2016].

27. Wilkinson J, Martinez A, Chen P et al. Four-year results using balloon-based brachytherapy to deliver accelerated partial breast irradiation with a 2-day dose fractionation schedule. Brachytherapy 2012; 11: 97-104.

28. Pignol J, Caudrelier J, Crook J et al. Report on the clinical outcomes of permanent seed breast implant for early-stage breast cancer. Int J Radiat Oncol Biol Phys 2015; 93: 614-621.

29. Vaidya J, Wenz F, Bulsara M et al. Risk-adapted targeted intraoperative radiotherapy versus whole-breast radiotherapy for breast cancer: 5-year results for local control and overall survival from TARGIT-A randomized trial. Lancet 2014; 33: 603-613.

30. Veronesi U, Orecchia R, Maisonneuve P et al. Intraoperative radiotherapy versus external radiotherapy for early breast cancer (ELIOT): a randomized controlled equivalence trial. Lancet Oncol 2013; 14: 1269-1277. 